\title{
Anti-carcinogenic effect of a new analogue 4'-chloroflavanone from flavanone in human breast cancer cells
}

\author{
EUN JEONG CHOI ${ }^{1}$, JAE IN LEE ${ }^{1,2}$ and GUN-HEE KIM ${ }^{1,3}$ \\ ${ }^{1}$ Plant Resources Research Institute and Departments of ${ }^{2}$ Chemistry and ${ }^{3}$ Food and Nutrition, \\ Duksung Women's University, 419 Ssangmun-dong, Tobong-ku, Seoul 132-714, Korea
}

Received September 11, 2009; Accepted November 6, 2009

\section{DOI: $10.3892 /$ ijmm_00000344}

\begin{abstract}
We investigated the antiproliferative effects of synthetic flavanone derivatives using an MTT assay in MCF-7 and MDA-MB-453 cells. When cells were treated with synthetic flavanone derivatives in concentrations ranging from 1 to $200 \mu \mathrm{M}$ for $48 \mathrm{~h}$, cell growth decreased at concentrations $>50 \mu \mathrm{M}$. 4'-Chloroflavanone is more potent than flavanone among the synthetic flavanone derivatives. Exposure to 4 '-chloroflavanone at $50 \mu \mathrm{M}$ for $48 \mathrm{~h}$ caused cell cycle arrest in both MCF-7 and MDA-MB-453 cells. In addition, when 4'-chloroflavanone caused G1/S phase arrest, a decrease in CDK4 and cyclin D, together with an increase in $\mathrm{p} 21^{\text {Cip1 }}$, was observed in the cells. The $\mathrm{p} 21^{\mathrm{Cip} 1}$ is a downstream target of $\mathrm{p} 53$ that may be affected by the activation of $\mathrm{p} 53$ by 4 '-chloroflavanone. These results indicate that activation of p53 played some role in 4'-chloroflavanone-induced cell cycle arrest of human breast cancer cells. 4'-Chloroflavanone increased cytochrome c expression and decreased the expression of caspase-3, but did not change the expression of Bcl-2 and Bax. Activation of cytochrome $\mathrm{c}$ and its downstream target, caspase- 3 , is suggested to be an important inducer of the apoptosis process by 4'-chloroflavanone. 4'-Chloroflavanone inhibits cell proliferation through $\mathrm{G} 1 / \mathrm{S}$ phase disruption and may induce apoptosis. Based on our findings, we propose that 4'-chloro-flavanone is useful as an anticancer drug.
\end{abstract}

\section{Introduction}

Flavonoids are a group of phytochemicals that include flavones, flavonols, flavanones, and isoflavones and are usually present almost exclusively in the form of $\beta$-glycosides in plants. Because of their benefits to human health, the biological activity of flavonoids has been extensively examined in terms of their anti-inflammatory, -tumor, and -oxidant capacity (1-4). These biological activities seem to be associated with their structure.

Correspondence to: Professor Gun-Hee Kim, Plant Resources Research Institute, Duksung Women's University, 419 Sangmundong, Tobong-ku, Seoul 132-714, Korea

E-mail: ghkim@duksung.ac.kr

Key words: apoptosis, 4'-chloroflavanone, cell proliferation, flavanone, synthetic derivatives
The structure-activity relationship (SAR) of flavonoids has been shown to depend on their chemical structure, including the degree of hydroxyl substitution and presence of a catecholtype $\mathrm{B}$ ring $(5,6)$.

On the other hand, flavonoids have been reported to possess prooxidant activity, depending on the experimental conditions $(7,8)$. Although the prooxidant activity of flavonoids was previously considered to be toxic causing cell death, similar properties could contribute to tumor apoptosis and cancer chemotherapy (9).

Flavanone, as an important type of naturally occurring flavonoid compound, is specifically present in citrus fruits and juices and constitutes a relatively high proportion of total daily flavonoid intake $(10,11)$.

Currently, one possible strategy for cancer prevention and treatment is the examination of natural compounds for nontoxic chemopreventive agents. For these reasons, interest in the use of flavanone as a cancer preventive or cancer treatment drug is growing.

To search for a new candidate drug for the treatment of human breast cancer, we evaluated the anti-proliferative activity and cell cycle arrest effects of flavanone and its synthetic derivatives. In addition, we aimed to elucidate the apoptosis induction pathway of flavanone and its synthetic derivatives. Our results show that flavanone and its synthetic derivatives have strong anti-proliferative effects on human breast cancer cells by way of p53-mediated apoptosis and the induction of cell cycle arrest at the G1 phase.

\section{Materials and methods}

Synthetic flavanone derivatives. Flavanone derivatives used in the present study were synthesized from 2'-hydroxyacetophenones at the Department of Chemistry, Duksung Women's University (South Korea). The subsequent reaction of lithium enolates, generated from 2'-hydroxyacetophenones and LDA, with benzaldehydes in THF afforded 1-(2'-hydroxyphenyl)-1oxo-propan-3-phenyl-3-ols, were cyclodehydrated with diethyl azodicarboxylate and $\mathrm{Ph} 3 \mathrm{P}$ in $\mathrm{CH}_{2} \mathrm{Cl}_{2}$ to give flavanones in high yields. These compounds were purified by recrystallization followed by silica-gel column chromatography when recrystallization was inefficient. Compound integrity and purity were confirmed by thin-layer chromatography (TLC) and nuclear magnetic resonance (NMR, Bruker DPX-300, German), gas chromatography (GC-mass, Agilent 
Table I. Inhibition of cell proliferation (inhibition $\%$ at 50 and $100 \mu \mathrm{M}$, respectively) and cytotoxicity of various synthetic flavanone derivatives in human breast cancer MCF-7 cells, after $48 \mathrm{~h}$ exposure.

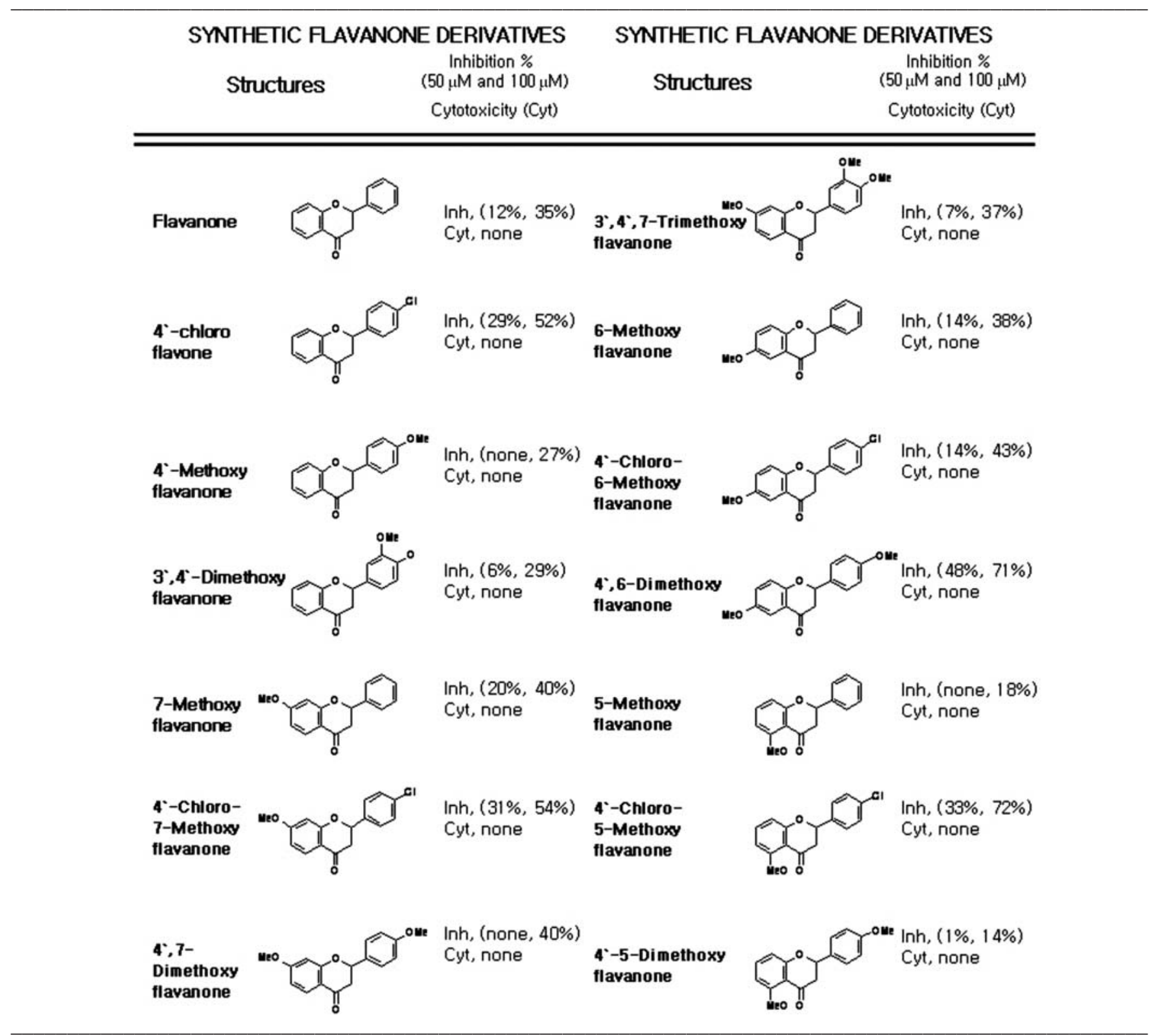

MCF-7 cells were exposed to synthetic flavanone derivatives at various concentrations (ranging from 1 to $200 \mu \mathrm{M}$ ) for $48 \mathrm{~h}$.

$6890 \mathrm{~N}$, USA) and all compounds included in the study were at least $95 \%$ pure. All compounds were dissolved in dimethyl sulfoxide (DMSO, final concentration $0.2 \%$ in medium).

Cell culture and treatment of synthetic flavanone derivatives. MCF-7 and MDA-MB-453 cells were purchased from the Korean Cell Line Bank (KCLB, Korea). Cells were routinely maintained in RPMI-1640 [Invitrogen (Molecular Probes), Gibco, Carlsbad, USA], supplemented with $10 \%$ FBS and antibiotics $(50 \mathrm{U} / \mathrm{ml}$ of penicillin and $50 \mu \mathrm{g} / \mathrm{ml}$ streptomycin, Gibco) at $37^{\circ} \mathrm{C}$ in a humidified atmosphere containing $5 \% \mathrm{CO}_{2}$. In cell proliferation and cytotoxicity experiments, cells were treated with various synthetic flavanone derivatives ranging from 1 to $200 \mu \mathrm{M}$ for $48 \mathrm{~h}$. In cell cycle analysis and apoptosis assay, cells were treated with either flavanone or 4'-chloroflavanone at $50 \mu \mathrm{M}$ for $48 \mathrm{~h}$.
Determination of cell proliferation and cytotoxicity. Cell proliferation and cytotoxicity was determined using the MTT and $\mathrm{LDH}$ release assay, respectively. $\mathrm{LDH}$ release was determined by formation of NADH (absorbance $340 \mathrm{~nm}$ ). Protein concentration was determined using a Bradford protein assay kit II (Bio-Rad Laboratories, CA, USA).

Cell cycle distribution. For cell cycle analysis, after cells were fixed in absolute ethanol, RNase A $(20 \mu \mathrm{g} / \mathrm{ml}$ final concentration) and propidium iodide staining solution $(50 \mu \mathrm{g} / \mathrm{ml}$ final concentration) were added to cells and incubated for $30 \mathrm{~min}$ at $37^{\circ} \mathrm{C}$ in the dark. Cells were analyzed by a FACS Calibur instrument (BD Biosciences, San Jose, USA) equipped with CellQuest 3.3 software. ModFit LT 3.1 trial cell cycle analysis software was used to determine the percentage of cells in the different phases of the cell cycle. 
Table II. Inhibition of cell proliferation (inhibition $\%$ at 50 and $100 \mu \mathrm{M}$, respectively) and cytotoxicity of various synthetic flavanone derivatives in human breast cancer MDA-MB-453 cells, after $48 \mathrm{~h}$ exposure.

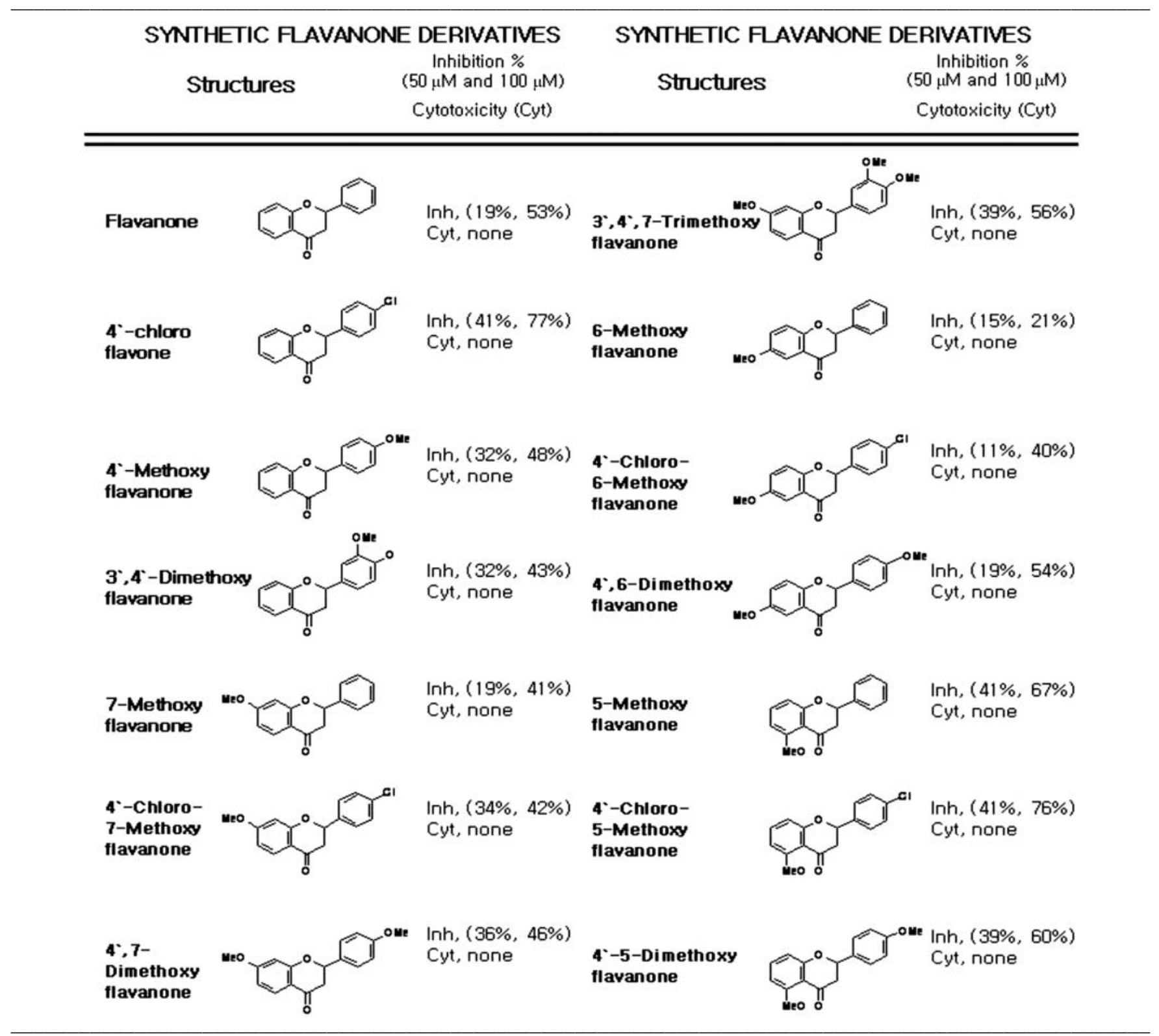

MDA-MB-453 cells were exposed to synthetic flavanone derivatives at various concentrations (ranging from 1 to $200 \mu \mathrm{M}$ ) and incubated for $48 \mathrm{~h}$.

Immunoblotting assay. Cells were lysed in RIPA buffer (1\% NP-40, $150 \mathrm{mM} \mathrm{NaCl}, 0.05 \%$ DOC, $1 \%$ SDS, $50 \mathrm{mM}$ Tris, $\mathrm{pH}$ 7.5) and their protein concentration was determined by Bradford protein assay kit II (Bio-Rad). Samples (25 $\mu \mathrm{g}$ protein/well) were separated by $10 \%$ SDS-polyacrylamide gel. Antibodies against CDK4, CDK6, cyclin D, p21 Cip1, p53, Bcl-2, Bax, cytochrome c, precursor caspase-3, and B-actin were purchased from Santa Cruz (Santa Cruz Biotechnology, Inc., USA) and used to probe the separate membranes. The specific protein bands were detected by Opti-4CN Substrate kit (Bio-Rad).

Statistical analyses. The data were expressed as percent compared with vehicle-treated control cells, which were arbitrarily assigned $100 \%$. Data were analyzed by one-way analysis of variance followed by Dunnett's multiple comparison test (Sigma Stat, Jandel, San Rafael, USA). For all comparisons, differences were considered statistically significant at $\mathrm{P}<0.05$.

\section{Results}

Inhibition of cell proliferation by synthetic flavanone derivatives. Synthetic flavanone derivatives affect the proliferation and cytotoxicity of human breast cancer cells (MCF-7 and MDA-MB-453; Tables I and II). Cell proliferation and cytotoxicity were determined by measuring MTT and the release of lactate dehydrogenase $(\mathrm{LDH})$, respectively. More than $48 \mathrm{~h}$ of exposure to most synthetic flavanone derivatives caused decreased cell proliferation at concentrations $>50 \mu \mathrm{M}$. Flavanone was the most potent anti-proliferative agent, with significant decreases in cell proliferation at flavanone concentrations of $50 \mu \mathrm{M}$ in MCF-7 cells and $5 \mu \mathrm{M}$ in MDA- 

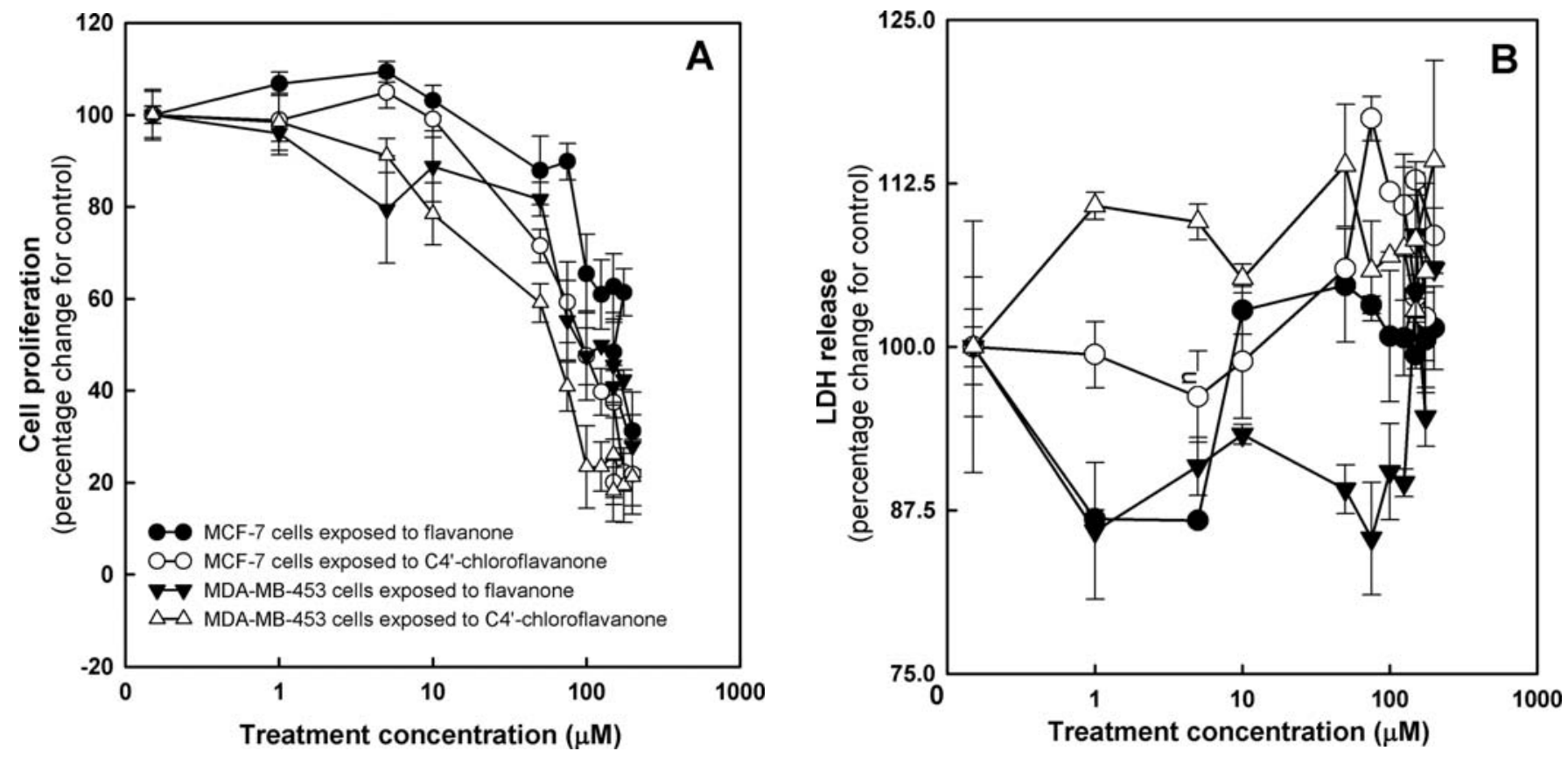

Figure 1. Cell proliferation (A) and cytotoxicity (B) on human breast cancer cells exposed to either flavanone or 4'-chloroflavanone. Cells were exposed to either flavanone or 4'-chloroflavanone at various concentrations (ranging from 1 to $200 \mu \mathrm{M}$ ) and incubated for $48 \mathrm{~h}$. All data are reported as the percentage change in comparison with the vehicle-only group, which were arbitrarily assigned $100 \%$ viability.
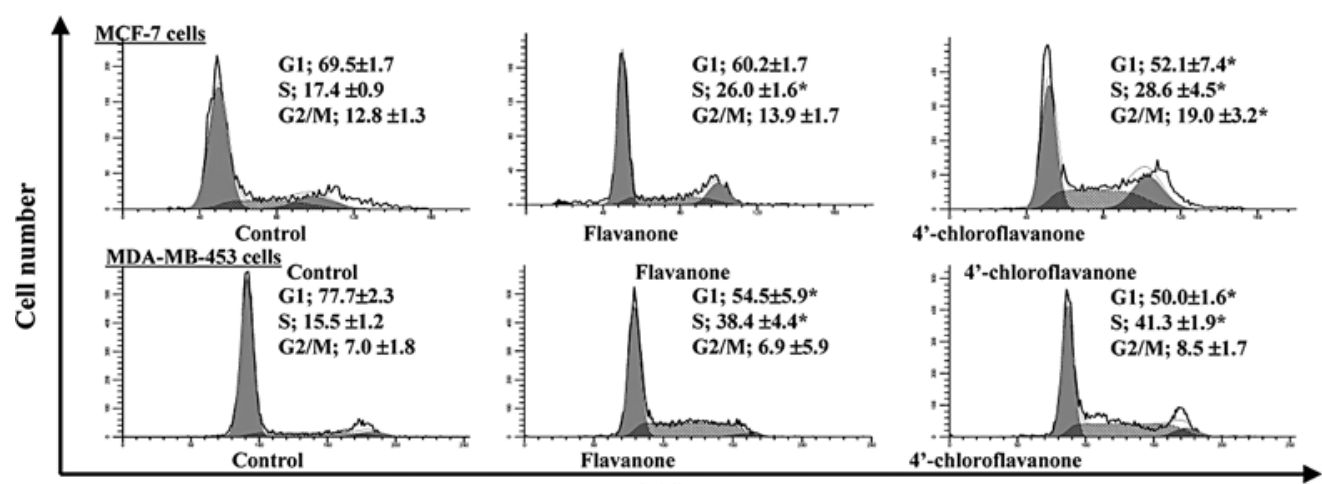

DNA content

Figure 2. Effect of 4'-chloroflavanone on cell cycle distribution of human cancer cells, compared with flavanone. Cells were exposed to either flavanone or 4 '-chloroflavanone at $50 \mu \mathrm{M}$ for $48 \mathrm{~h} .{ }^{*} \mathrm{P}<0.05$, significantly different from the vehicle-only group.

B-453 cells. At concentrations ranging from 1 to $50 \mu \mathrm{M}$, some synthetic flavanone derivatives did not lower cell proliferation. Some synthetic flavanones showed a trend toward increasing cell proliferation, although there was no statistical difference. In addition, treatment with synthetic flavanone derivatives for $48 \mathrm{~h}$ at concentrations up to $200 \mu \mathrm{M}$ did not show any evidence of cytotoxicity in either MCF-7 or MDAMB-453 cells.

Among the synthetic flavanone derivatives, 4'-chloroflavanone was more effective at preventing cell proliferation than flavanone was (Fig. 1A). That is, exposure to 4'-chloroflavanone decreased cell proliferation by 27.2 and $50.6 \%$ in MCF-7 and MDA-MB-453 cells, respectively, compared to exposure to flavanone at the same concentration. There was no evidence of cytotoxicity (Fig. 1B).

Cell cycle distribution by flavanone and 4'-chloroflavanone. Cell cycle arrest in both MCF-7 and MDA-MB-453 cells $48 \mathrm{~h}$ after exposure to either flavanone or 4'-chloroflavanone was observed at concentrations of $50 \mu \mathrm{M}$ (Fig. 2). After exposure to flavanone, the proportion of MCF-7 cells in the G1 phase decreased from 69.5 to $60.2 \%$, and the proportion of S-phase cells significantly increased from 17.4 to $26.0 \%$ compared with control cells. A significant decrease in the number of G1-phase cells was seen after 4'-chloroflavanone exposure at concentrations of $50 \mu \mathrm{M}(52.13 \%$ of the cell population compared with the control value of $69.5 \%$; $<<0.05)$. The proportion of MCF-7 cells in the S phase increased from 17.4 to $28.6 \%$, and the proportion of $\mathrm{G} 2 / \mathrm{M}$-phase cells decreased from 12.8 to $19.0 \%$. More pronounced results were obtained when MDA-MB-453 cells were treated with flavanone and 4'-chloroflavanone.

G1-phase-related protein expression by flavanone and 4 '-chloroflavanone. When cell cycle arrest was induced by treatment with either flavanone or 4'-chloroflavanone, we 

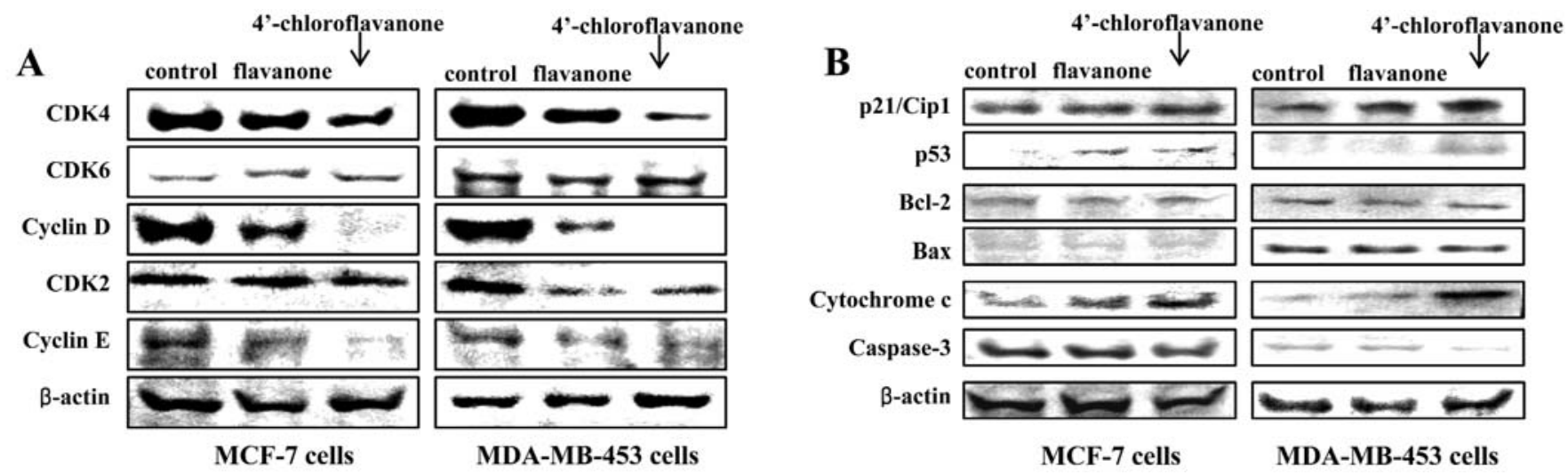

Figure 3. Effect of flavanone and 4'-chloroflavanone on G1-related regulator (A) and apoptosis related protein expression (B) of human cancer cells. Cells were exposed to either flavanone or 4'-chloroflavanone at $50 \mu \mathrm{M}$ for $48 \mathrm{~h}$.

observed the expression of cyclin D and cyclin-dependent kinase CDK4 and CDK6 (Fig. 3A). Expression of CDK4 and cyclin D was significantly decreased by 4'-chloroflavanone. Flavanone exposure caused a decrease in CDK4 and cyclin D, although the decrease was not as large as that observed after 4'-chloroflavanone exposure. CDK6 expression was not affected by either flavanone or 4'-chloroflavanone. Expression of p21 $1^{\text {Cip1 }}$, one of the CDK inhibitory subunits, was increased by both flavanone and 4'-chloroflavanone. Protein expression modulation by both flavanone and 4'-chloroflavanone showed similar patterns in both MCF-7 and MDA-MB-453 cells.

To investigate the apoptosis process induced by flavanone and 4'-chloroflavanone in MCF-7 and MDA-MB-453 cells, we assayed the protein expression of an apoptosis mediator (Fig. 3B). Activation of p53 was observed upon treatment with flavanone or 4'-chloroflavanone. Bcl-2 and Bax expression was not changed in either MCF-7 or MDA-MB-453 cells exposed to either flavanone or 4'-chloroflavanone. Cytochrome c was released by treatment with both flavanone and 4'-chloroflavanone, and the precursor caspase- 3 was affected by 4'-chloroflavanone.

\section{Discussion}

We found that synthetic flavanone derivatives have a significant inhibitory effect on the proliferation of two human breast cancer cell lines, MCF-7 and MDA-MB-453. In addition, treatment for $48 \mathrm{~h}$ with synthetic flavanone derivatives did not cause cytotoxicity in either MCF-7 or MDA-MB-453 cells.

We also investigated changes to the cell cycle in human breast cancer cell lines exposed to 4'-chloroflavanone compared to those exposed to flavanone at the same concentration. Both flavanone and 4'-chloroflavanone exposure resulted in the induction of cell cycle arrest. It has been reported that flavanone treatment also resulted in $\mathrm{G}_{1}$ and $\mathrm{G}_{2} / \mathrm{M}$ arrest in human prostate cancer PC3 cells (12). However, 4'-chloroflavanone appeared to be more potent at causing cell cycle arrest. 4'-Chloroflavanone treatment resulted in an increase in $\mathrm{p} 21^{\mathrm{Cip} 1}$ proteins and a significant down-regulation of CDK4/cyclin D expression. This may be an important molecular mechanism through which flavanone inhibits growth of cancer cells. The p21 Cip1, a downstream target of p53, could inhibit the G1-S-phase transition and result in G1-phase cell cycle arrest $(13,14)$.
The tumor suppressor gene p53 is regarded as a key factor in a balance between cell survival and cell death via regulation of both the $G_{1}$ and $G_{2} / M$ portions of the cell cycle (16). Activation of p53 in response to DNA damage led to cell cycle arrest and inhibition of cell proliferation (17-19). 4'-Chloroflavanone exposure increased p53 expression in both MCF-7 and MDA-MB-453 cells. Thus, we suggest that the activation of p53 plays a role in the 4'-chloroflavanone-induced cell cycle arrest of human breast cancer cells via p21 ${ }^{\text {Cipl }}$ induction.

Members of the Bcl-2 family of proteins are critical regulators of the apoptotic pathway, controlling mitochondrial permeability and cytochrome c expression $(20,21)$. These proteins consist of the major antiapoptotic family members, $\mathrm{Bcl}-\mathrm{x}(\mathrm{L})$ and $\mathrm{Bcl}-2$, and the major proapoptotic proteins, Bax and Bak. Modulation of Bcl-2 and Bax was not observed after exposure to 4'-chloroflavanone.

4'-Chloroflavanone induced the expression of cytochrome c and decreased the expression of a precursor of caspase- 3 . These results suggest that up-regulation of cytochrome c may be another molecular mechanism through which flavanone and 4'-chloroflavanone induce apoptosis (22). Release of cytochrome c from mitochondria to the cytoplasm is a key step in the initiation of apoptosis. As a downstream product of cytochrome c, caspases are critical mediators of the principle factors found in apoptotic cells. Among them, caspase- 3 is a frequently activated death protease, catalyzing the specific cleavage of many key cellular proteins $(23,24)$. Our results show that the activity of caspase- 3 was significantly increased in both MCF-7 and MDA-MB-453 cells after exposure to 4'-chloroflavanone. Activation of caspase-3 after the release of cytochrome $\mathrm{c}$ is therefore suggested to be an important determinant of apoptosis induced by 4'-chloroflavanone in human breast cancer cells. Except for caspase-3 modulation, flavanone displayed the same pattern as 4'-chloroflavanone in all processes. This may indicate that flavanone is less potent than 4'-chloroflavanone.

Based on our results, we suggest multiple pathways by which flavanone and its synthetic derivatives result in apoptotic cell death. Induction of p21 $1^{\mathrm{Cip} 1}$ in $4^{\prime}$-chloroflavanone-treated human breast cancer cells appeared to be caused by the cell cycle arrest at the G1/S phase. In addition, 4'-chloroflavanone induced p53 and cytochrome c release which were also correlated with apoptosis. Collectively, apoptosis by 4'-chloro- 
flavanone may be mediated via cell cycle arrest, modulation of the p53 pathway, and up-regulation of cytochrome c expression.

\section{Acknowledgements}

This work was supported by the National Research Foundation of Korea Grant funded by the Korean Government (KRF2008-005-J00601).

\section{References}

1. Walle T: Absorption and metabolism of flavonoids. Free Radic Biol Med 36: 829-837, 2004.

2. Kim HP, Son KH, Chang HW and Kang SS: Anti-inflammatory plant flavonoids and cellular action mechanisms. J Pharmacol Sci 96: 229-245, 2004

3. Kanadaswami C, Lee LT, Lee PP, et al: The antitumor activities of flavonoids. In Vivo 19: 895-909, 2005.

4. Cushnie TP and Lamb AJ: Antimicrobial activity of flavonoids. Int J Antimicrob Agents 26: 343-356, 2005.

5. Pouget C, Lauthier F, Simon A, et al: Flavonoids: structural requirements for antiproliferative activity on breast cancer cells. Bioorg Med Chem Lett 11: 3095-3097, 2001.

6. Lopez-Lazaro M: Flavonoids as anticancer agents: structureactivity relationship study. Curr Med Chem Anticancer Agents 2: 691-714, 2002.

7. Choi EJ, Chee KM and Lee BH: Anti- and prooxidant effects of chronic quercetin administration in rats. Eur J Pharmacol 482: 281-285, 2003.

8. Choi EJ, Kim GD, Chee KM and Kim GH: Effects of hesperetin on vessel structure formation in mouse embryonic stem (mES) cells. Nutrition 22: 947-951, 2006.

9. Kris-Etherton PM, Lefevre M, Beecher GR, Gross MD, Keen CL and Etherton TD: Bioactive compounds in nutrition and healthresearch methodologies for establishing biological function: the antioxidant and anti-inflammatory effects of flavonoids on atherosclerosis. Annu Rev Nutr 24: 511-538, 2004.

10. Ameer B, Weintraub RA, Johnson JV, Yost RA and Rouseff RL: Flavanone absorption after naringin, hesperidin, and citrus administration. Clin Pharmacol Ther 60: 34-40, 1996.

11. Pelt JL, Downer WA, Schoborg RV and McIntosh CA: Flavanone 3-hydroxylase expression in Citrus paradisi and Petunia hybrida seedlings. Phytochemistry 34: 435-444, 2003.
12. Deep J, Singh RP, Agarwal C, Kroll DJ and Agawal R: Constitutive activation of cyclin B1-associated cdc2 kinase overrides p53-mediated G2-M arrest. Cancer Res 60: 1053-1069, 2006.

13. Niculescu AB 3rd, Chen X, Smeets M, Hengst L, Prives C and Reed SI: Effects of p21(Cip1/Waf1) at both the G1/S and the G2/M cell cycle transitions: $p R b$ is a critical determinant in blocking DNA replication and in preventing endoreduplication. Mol Cell Biol 18: 629-643, 1998.

14. Bai F, Matsui T, Ohtani-Fujita N, Matsukawa Y, Ding Y and Sakai T: Promoter activation and following induction of the p21/WAF1 gene by flavone is involved in G1 phase arrest in A549 lung adenocarcinoma cells. FEBS Lett 437: 61-64, 1998.

15. May P and May E: Twenty years of $p 53$ research: structural and functional aspects of the p53 protein. Oncogene 18: 7621-7636, 1999.

16. Agarwal ML, Agarwal A, Taylor WR and Stark GR: p53 controls both the G2/M and the G1 cell cycle checkpoints and mediates reversible growth arrest in human fibroblasts. Proc Natl Acad Sci USA 92: 8493-8497, 1995.

17. Park M, Chae HD, Yun J, et al: Constitutive activation of cyclin B1-associated cdc2 kinase overrides p53-mediated G2-M arrest. Cancer Res 60: 542-545, 2000.

18. Lepik D, Jaks V, Kadaja L, Varv S and Maimets T: Electroporation and carrier DNA cause p53 activation, cell cycle arrest, and apoptosis. Anal Biochem 318: 52-59, 2003.

19. Parker MA, Anderson JK, Corliss DA, et al: Expression profile of an operationally-defined neural stem cell clone. Exp Neurol 194: 320-332, 2005.

20. Oakea SA, Lin SS and Bassik MC: The control of endoplasmic reticulum-initiated apoptosis by the BCL-2 family of proteins. Curr Mol Med 6: 99-109, 2006.

21. Van Delft MF and Huang DC: How the Bcl-2 family of proteins interact to regulate apoptosis. Cell Res 16: 203-213, 2006.

22. Jiang $X$ and Xang X: Cytochrome C-mediated apoptosis. Annu Rev Biochem 73: 87-106, 2004.

23. Porter AG and Janicke RU: Emerging roles of caspase-3 in apoptosis. Cell Death Differ 6: 99-104, 1999.

24. Abu-Qare AW and Abou-Donia MB: Biomarkers of apoptosis: release of cytochrome $\mathrm{c}$, activation of caspase-3, induction of 8-hydroxy-2'-deoxyguanosine, increased 3-nitrotyrosine, and alteration of p53 gene. J Toxicol Environ Health B Crit Rev 4: 313-332, 2001. 